\title{
Direct Observation of Adsorption Sites of Protein Impurities and Their Effects on Step Advancement of Protein Crystals ${ }^{\dagger}$
}

\author{
Alexander E. S. Van Driessche, ${ }^{\ddagger}$ Gen Sazaki, ${ }^{*}, \$, \mathbb{\Perp}$ Guoliang Dai, ${ }^{\perp}$ Fermín Otálora, ${ }^{\ddagger}$ \\ Jose A. Gavira, ${ }^{*}$ Takuro Matsui," Izumi Yoshizaki, ${ }^{\#}$ Katsuo Tsukamoto, ${ }^{\nabla}$ and \\ Kazuo Nakajima ${ }^{\S}$
}

\begin{abstract}
Laboratorio de Estudios Crystalográficos, IACT, CSIC-U.Granada, P.T. Ciencias de la Salud, Avenida del conocimiento s/n, 18100 Armilla (Granada), Spain, Institute for Materials Research, Tohoku University, 2-1-1 Katahira, Aoba-ku, Sendai 980-8577, Japan, National Microgravity Laboratory, Institute of Mechanics, Chinese Academy of Science, 15 bei Si Huan Xi Road, Beijing 100080, China, New Technology Laboratory, Merck Ltd. Japan, 4084 Nakatsu, Aikawa-machi, Aiko-gun, Kanagawa 243-0303, Japan, ISS Science Project Office, Institute of Space and Astronautical Science, Japan Aerospace Exploration Agency, 2-1-1 Sengen, Tsukuba 305-8505, Japan, and Graduate School of Science, Tohoku University, Aza-Aoba, Aramaki, Sendai 980-8578, Japan
\end{abstract}

Received June 24, 2008; Revised Manuscript Received May 7, 2009

\begin{abstract}
We measured noninvasively step velocities of elementary two-dimensional (2D) islands on $\{110\}$ faces of tetragonal lysozyme crystals, under various supersaturations, by laser confocal microscopy combined with differential interference contrast microscopy. We studied the correlation between the effects of protein impurities on the growth of elementary steps and their adsorption sites on a crystal surface, using three kinds of proteins: fluorescent-labeled lysozyme (F-lysozyme), covalently bonded dimers of lysozyme (dimer), and a $18 \mathrm{kDa}$ polypeptide $(18 \mathrm{kDa})$. These three protein impurities suppressed the advancement of the steps. However, they exhibited different supersaturation dependencies of the suppression of the step velocities. To clarify the cause of this difference, we observed in situ the adsorption sites of individual molecules of F-lysozyme and fluorescent-labeled dimer (F-dimer) on the crystal surface by single-molecule visualization. We found that F-lysozyme adsorbed preferentially on steps (i.e., kinks), whereas F-dimer adsorbed randomly on terraces. Taking into account the different adsorption sites of F-lysozyme and F-dimer, we could successfully explain the different effects of the impurities on the step velocities. These observations strongly suggest that 18 $\mathrm{kDa}$ also adsorbs randomly on terraces. Seikagaku lysozyme exhibited a complex effect that could not alone be explained by the two major impurities (dimer and $18 \mathrm{kDa}$ ) present in Seikagaku lysozyme, indicating that trace amounts of other impurities significantly affect the step advancement.
\end{abstract}

\section{Introduction}

To understand the possible factors affecting the quality of protein crystals, it is indispensable to clarify the effects of impurities, since impurities can significantly affect the growth kinetics, ${ }^{1-11}$ morphology, ${ }^{12-15}$ lattice disorder, ${ }^{16}$ and hence quality of protein crystals. ${ }^{17-24}$ Generally, impurities are considered to exhibit their effects on the growth process after they adsorb on a crystal surface. Thus, to fully comprehend the mechanisms of impurity effects, one has to observe in situ both (1) dynamics of elementary steps and (2) adsorption of impure molecules on a crystal surface, at a molecular level.

To our knowledge, only a few such observations were made so far. By atomic force microscopy (AFM), Nakada and coworkers $^{9}$ observed elementary growth steps and individual molecules of covalently bonded dimer of lysozyme (hereafter dimer) at the same time, on a $\{101\}$ face of a tetragonal lysozyme crystal. They were able to visualize dimer molecules absorbed on the crystal surface by AFM because of

\footnotetext{
Originally submitted as part of the special issue (Cryst. Growth Des. $\mathbf{2 0 0 8}$ $8(12)$ ) on the 12th International Conference on the Crystallization of Biological Macromolecules, Cancun, Mexico, May 6-9, 2008.

* Corresponding author. E-mail: sazaki@lowtem.hokudai.ac.jp.

IACT, CSIC-U.Granada.

Institute for Materials Research, Tohoku University.

${ }^{\perp}$ National Microgravity Laboratory.

"Merck Ltd. Japan.

\# Institute of Space and Astronautical Science, Japan Aerospace Exploration Agency.

${ }^{\nabla}$ Graduate School of Science, Tohoku University.

"I Present address: The Institute of Low Temperature Science, Hokkaido University, N19-W8, Sapporo 060-0819, Japan.
}

their bigger size compared to monomeric lysozyme molecules. They revealed that dimer molecules adsorb randomly on terraces and suppress the advancement of elementary growth steps. On surfaces of ferritin and apoferritin crystals, Yau and co-workers also succeeded in visualizing, by AFM, individual impure protein molecules with different molecular sizes. $^{25,26}$ Although Yau and co-workers did not discuss impurity effects on step advancement, their works are excellent examples of visualization of impure molecules on a crystal surface.

However, to clarify the general picture of impurity effects, one has to visualize impurity molecules irrespective of the size of these molecules. For example, in the case of a protein impurity molecule whose molecular surface is slightly different from that of a solute protein molecule (e.g., fluorescent-labeled lysozyme: F-lysozyme), ${ }^{5}$ the spatial resolution of AFM is not sufficient to distinguish these impurity molecules from the solute molecules. Therefore, to observe individual impurity molecules adsorbed on a crystal surface, a different methodology is necessary. To our knowledge, one of the most promising alternatives is single-molecule visualization by total internal reflection fluorescence microscopy, ${ }^{27-30}$ by which one can track the movement of individual molecules with a fluorescent label. Very recently, we succeeded in observing individual molecules of fluorescent-labeled lysozyme (F-lysozyme) at an interface between a solution and a lysozyme crystal, using single-molecule visualization of a thin-solution-layer type. ${ }^{31,32}$ We reported that strong interactions with the crystal surface significantly condense F-lysozyme molecules at the interface and make their diffusion 


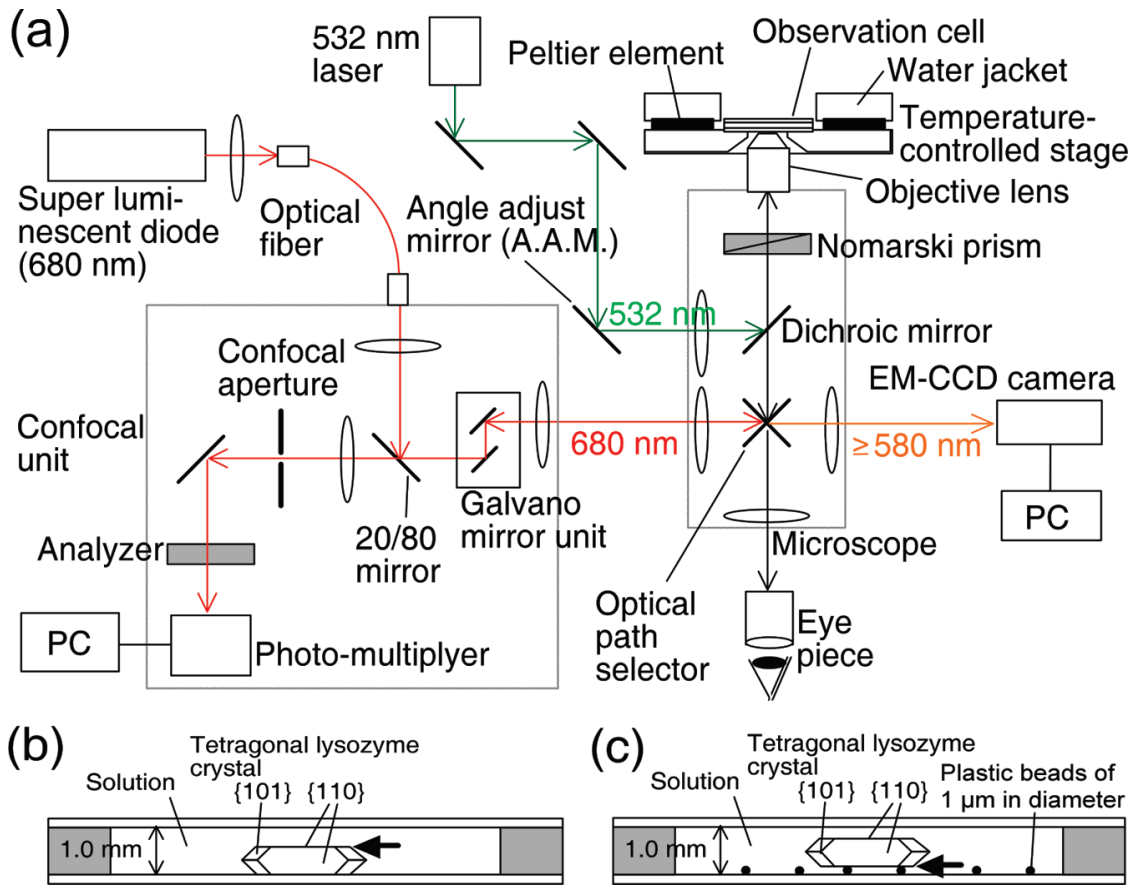

Figure 1. Schematic illustrations of an experimental setup: (a) a LCM-DIM and single-molecule visualization system and a temperature-controlled stage; cross-sectional views of observation cells used for in situ observation by LCM-DIM (b) and single-molecule visualization of a thin-solutionlayer type (c). Bold arrows indicate the crystal-solution interfaces at which in situ observations were carried out.

$4-5$ orders of magnitude slower than in a bulk solution. ${ }^{31}$ In addition, we obtained direct evidence that the molecules are gradually immobilized on the crystal surface, and a certain induction period exists before the gradual immobilization starts. ${ }^{32}$

To reveal impurity effects on the growth of a crystal, the response of step advancement to adsorbed impurities should also be measured in situ. To measure step velocity, we chose optical microscopy, since AFM disturbs the solute-depletion layer around a growing crystal in particular under high supersaturation range. ${ }^{33}$ Several optical microscopy techniques, such as phase contrast microscopy, ${ }^{10,34,35}$ laser confocal microscopy combined with differential interference contrast microscopy (LCM-DIM), ${ }^{11,36-38}$ and phase shift interferometry ${ }^{39}$ are available to observe advancement of individual elementary growth steps on a growing protein crystal completely noninvasively. Dai and coworkers $^{32}$ very recently demonstrated that by using LCMDIM and single-molecule visualization, steps and F-lysozyme molecules on a crystal surface can be visualized in the same field of view.

Thus, in this study, we used LCM-DIM and single-molecule visualization of the thin-solution-layer type, and simultaneously observed elementary growth steps on $\{110\}$ faces and fluorescentlabeled protein molecules adsorbed on the $\{110\}$ faces of tetragonal lysozyme crystals. First, we measured step velocities under the presence of various protein impurities to quantify the impurity effects on the dynamics of elementary growth steps. As protein impurities, we used F-lysozyme, dimer, and $18 \mathrm{kDa}$ protein (the last two are major impurities of Seikagaku lysozyme).$^{40}$ Second, we used F-lysozyme and fluorescentlabeled dimer (F-dimer) for the observation of adsorption sites of these proteins on the crystal surface. From these in situ observations, we tried to clarify directly the relation between the effects of the protein impurities on the advancement of elementary steps and the adsorption sites of impurities on the crystal surface, for the first time.

\section{Experimental Methods}

2.1. Experimental Setup for in Situ Observation. $\{110\}$ faces of tetragonal lysozyme crystals were observed in situ by LCM-DIM and single-molecule visualization of the thin-solution-layer type. Figure 1a shows a schematic illustration of the experimental setup. A confocal system (FV300, Olympus) and a diagonal illumination system using an objective were constructed on an inverted optical microscope (IX70, Olympus). A $20 \times$ objective (LUCPlan Fl 20x, Olympus) and a super luminescent diode (Amonics Ltd., model ASLD68-050-B-FA: $680 \mathrm{~nm}$ ), whose coherent length and full-width at half-maximum were about 10 $\mu \mathrm{m}$ and $23 \mathrm{~nm}$, were used for LCM-DIM measurements of step velocities. The use of the super luminescent diode was beneficial for removing interference fringes from LCM-DIM images. A $60 \times$ oilimmersion objective (PlanApo 60xO TIRFM3, Olympus) was used for the observations of the adsorption sites of individual F-lysozyme and F-dimer molecules on the crystal surface. These fluorescent labeled molecules were illuminated with a $532 \mathrm{~nm}$ laser, and emission at $\geq 580$ $\mathrm{nm}$ was recorded, at a frame rate of $100 \mathrm{~ms}$, with an electron multiplying charge-coupled device camera (EM-CCD: DV887, Andor Technology). The $60 \times$ objective was used to observe the same field of view by LCMDIM and single-molecule visualization.

Figure $1 \mathrm{~b}$ presents a sectional view of an observation cell $(1 \times 10$ $\times 20 \mathrm{~mm}^{3}$ ) used for step velocity measurements. The observation cell was made of two glass plates of $0.17 \mathrm{~mm}$ thickness separated by 1 $\mathrm{mm}$ polystyrene spacers. The bold arrow in Figure $1 \mathrm{~b}$ indicates the interface at which step velocities were measured by LCM-DIM. A sectional view of an observation cell $\left(1 \times 10 \times 20 \mathrm{~mm}^{3}\right)$ for the singlemolecule visualization experiments is shown schematically in Figure 1c. A tetragonal lysozyme crystal was placed on $1 \mu \mathrm{m}$ spacer beads adsorbed on a bottom glass plate. ${ }^{31,32}$ The solution-crystal interface (bold arrow in Figure 1c) was illuminated by a laser beam tilted almost parallel to the crystal surface to avoid an increase in the background intensity of the fluorescent light. Other details of the observations are explained in our previous works. ${ }^{11,31,32,36,37}$

2.2. Step Velocity Measurement and Adsorption Site Observation. Tetragonal crystals of model protein hen egg-white lysozyme were grown at $20.0 \pm 0.1{ }^{\circ} \mathrm{C}$ from a solution containing $70 \mathrm{mg} / \mathrm{mL}$ Seikagaku lysozyme (98.5\% purity: $6 \times$ recrystallized, Seikagaku Co.), $25 \mathrm{mg} /$ $\mathrm{mL} \mathrm{NaCl}$, and $50 \mathrm{mM}$ sodium acetate ( $\mathrm{pH} 4.5$ ) buffer. After the seed crystals were transferred to the observation cell (Figure 1b), the solution 
inside the cell was replaced with a supersaturated solution of $99.99 \%$ pure lysozyme (Maruwa Food Industries, Inc.), and the seed crystals were incubated for 5-6 h until the surfaces of the seed crystals were covered with newly grown layers of $99.99 \%$ pure lysozyme. We did not grow crystals only using highly purified protein because this purified lysozyme sample was very expensive. Even so, these "mixed" crystals were considered "pure" for studying growth kinetics since crystal surfaces prepared in this way showed no significant impurity effect on two-dimensional (2D) nucleation kinetics. ${ }^{11}$ Before starting the step velocity measurements, the solution inside the cell was replaced again with a $99.99 \%$ pure lysozyme solution containing a given concentration of an impure protein and was incubated for $24 \mathrm{~h}$ (no significant depletion of solute protein occurred during the incubation period). The observation cell was then set on a temperature-controlled stage with Peltier elements, and 2D islands formed on the $\{110\}$ faces were observed by LCM-DIM. The temperature of the observation cell was changed at given time intervals to change the supersaturation of the lysozyme solution inside the cell: the accuracy of the temperature control was $\pm 0.1{ }^{\circ} \mathrm{C}$. The solubility was calculated from the data reported by Sazaki et al. ${ }^{41}$ Step velocities $v$ were represented as follows: ${ }^{42}$

$$
v=\Omega \beta\left(C-C_{\mathrm{e}}\right)
$$

where $\Omega$ is the volume of one lysozyme molecule inside a tetragonal crystals $\left(=3 \times 10^{-20} \mathrm{~cm}^{3}\right),{ }^{43} \beta$ is the step kinetic coefficient, $C$ is the bulk concentration of lysozyme and $C_{\mathrm{e}}$ is the solubility at a given temperature. In our previous work, we have shown that eq 1 is a useful approximation for step velocities measured in the temperature range of $18-26^{\circ} \mathrm{C}$ (i.e., the step kinetic coefficient is constant for this temperature range). ${ }^{44}$ Observations were carried out in the supersaturation range $C-C_{\mathrm{e}}=0-45 \mathrm{mg} / \mathrm{mL}$.

The observation cell used for single-molecule visualization of adsorption sites of F-lysozyme and F-dimer molecules (Figure 1c) was prepared according to the recipe reported in our previous works. ${ }^{31,32}$ The lysozyme concentration used for these experiments was $27 \mathrm{mg} /$ $\mathrm{mL}$, at which concentration the step velocity of elementary steps was negligibly small at $24.5^{\circ} \mathrm{C}\left(C_{\mathrm{e}} \approx 27 \mathrm{mg} / \mathrm{mL}\right)$. Individual molecules of F-lysozyme and F-dimer (both used in a concentration of $0.1 \mathrm{nM}$ ) were observed in situ on $\{110\}$ faces by single-molecule visualization, as reported previously. ${ }^{31,32}$ Before and after the single-molecule observation, the same field of view was observed by LCM-DIM to check that steps did not move significantly during the single-molecule observation. From the comparison between the images taken by LCM-DIM and single-molecule visualization, adsorption sites of F-lysozyme and F-dimer were determined.

2.3. Protein Impurities. Three kinds of protein impurities were used for the step velocity measurements by LCM-DIM. One is fluorescent-labeled lysozyme (F-lysozyme), which was prepared according to the recipe reported by Matsui and co-workers. ${ }^{5}$ Only the $\varepsilon$-amino group of the $\mathrm{N}$-terminal lysine of hen egg-white lysozyme is specifically labeled with a fluorescent reagent, tetramethylrhodamine5-isothiocyanate (5-TRITC, MW $=443 \mathrm{~g} / \mathrm{mol}$, Molecular Probes $\mathrm{T}-1480),{ }^{5}$ by optimizing reaction conditions (details were described in our previous paper ${ }^{5}$ ). The two other impurities are covalently bonded dimer of lysozyme (dimer) and a $18 \mathrm{kDa}$ polypeptide (18 kDa), which were purchased from Maruwa Food Industries, Inc. Both dimer and $18 \mathrm{kDa}$ are known as the major impurities present in Seikagaku lysozyme (identity of $18 \mathrm{kDa}$ is still unclear). ${ }^{40}$ Following the classification of impurity proteins proposed by McPherson, ${ }^{45} \mathrm{~F}$ lysozyme and dimer are microheterogeneous impurities of lysozyme, and $18 \mathrm{kDa}$ is a macroheterogeneous impurity. Also Seikagaku lysozyme (98.5\% purity) was used for the measurements. Step velocity measurements in the presence of protein impurities were carried out in the supersaturation range $C-C_{\mathrm{e}}=0-31 \mathrm{mg} / \mathrm{mL}$. Since stock solutions of protein impurities could not be concentrated because of aggregation, it was not possible to prepare a lysozyme solution with $C-C_{\mathrm{e}}>31$ $\mathrm{mg} / \mathrm{mL}$ and still keeping a certain relative concentration of impurity to lysozyme.

For single-molecule observations, dimer was also chemically labeled with 5-TRITC according to the same recipe ${ }^{5}$ (hereafter fluorescentlabeled dimer is called F-dimer). In the case of F-dimer, the precise identification of the position at which 5-TRITC was labeled was difficult to achieve, because of the very limited amount of dimer available for
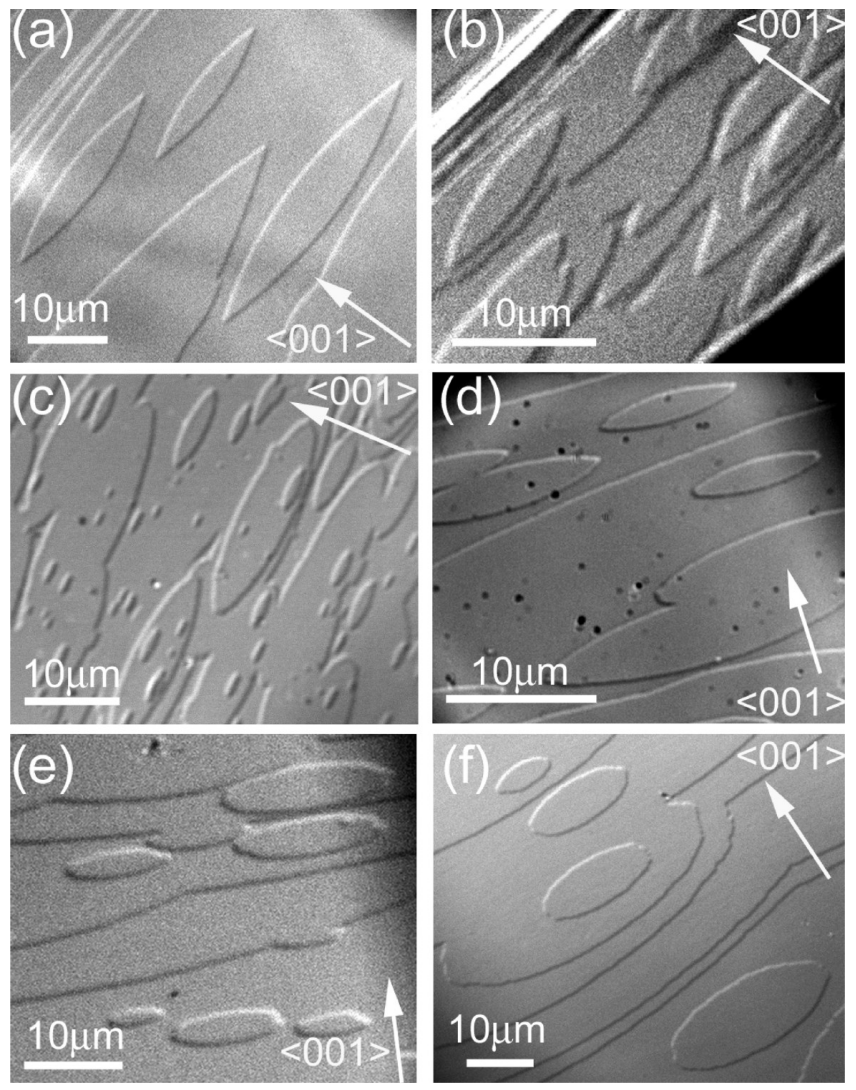

Figure 2. Photomicrographs of $2 \mathrm{D}$ islands on $\{110\}$ faces of tetragonal lysozyme crystals taken by LCM-DIM. In $(\mathrm{a}-\mathrm{e})$, all crystals were grown from $99.99 \%$ purity lysozyme solutions of $40 \mathrm{mg} / \mathrm{mL}$. Protein impurities added intentionally: (a) none, (b) 0.1 wt \% F-lysozyme, (c) $0.5 \mathrm{wt} \%$ dimer, (d) $1.0 \mathrm{wt} \% 18 \mathrm{kDa}$, and (e) $0.5 \mathrm{wt} \%$ dimer and 1.0 wt $\% 18 \mathrm{kDa}$. In (f), a crystal grown from a Seikagaku lysozyme (98.5\% purity) solution of $40 \mathrm{mg} / \mathrm{mL}$ is shown. Other growth conditions: 25 $\mathrm{mg} / \mathrm{mL} \mathrm{NaCl}$, in $50 \mathrm{mM}$ sodium acetate $(\mathrm{pH} 4.5)$, at $20.0-23.0{ }^{\circ} \mathrm{C}$.

the experiments, in addition to the unknown molecular structure of dimer. However, since a dimer molecule has twice the number of amino groups as a monomer and we used a dimer solution of the same proteinweight concentration (1/2 molar concentration) as the monomer solution, then it is reasonable to assume that one amino group of one dimer molecule was chemically modified with one fluorescent label. Since the available amount of $18 \mathrm{kDa}$ was too small, no fluorescent-labeled $18 \mathrm{kDa}$ molecules were prepared.

The purity level of the Maruwa sample $(99.99 \%)$ was verified by sodium dodecylsulfate polyacrylamide gel electrophoresis (SDS-PAGE) with enhanced silver staining (detection limit $\leq 0.01 \%$ ), and no trace of impurity (18 kDa or Dimer) could be found; also no significant shift was found in the purity level for storage times of several months at 4 ${ }^{\circ} \mathrm{C}$. Impurity contents of the Seikagaku sample (98.5\%) was also confirmed by SDS-PAGE: the Seikagaku sample contained $0.5 \%$ dimer and $1.0 \% 18 \mathrm{kDa}$, as reported by Thomas and co-workers. ${ }^{7}$

\section{Results and Discussion}

3.1. Impurity Effects on Step Velocities. We observed in situ the impurity effects of F-lysozyme, dimer, $18 \mathrm{kDa}$, and impurities included in Seikagaku lysozyme (98.5\% purity) on the morphology of elementary 2D islands on $\{110\}$ faces of tetragonal lysozyme crystals by LCM-DIM. As shown in Figure 2, 2D islands with elementary step height $(5.6 \mathrm{~nm})^{46-48}$ could be clearly visualized by LCM-DIM. ${ }^{11,33,36,37,44}$ The $2 \mathrm{D}$ islands formed in a $99.99 \%$ purity solution (Figure 2 a) are lens-shaped with sharp tips. This shape results from an intrinsic anisotropy in the step velocities, $\langle 001\rangle$ being the slow direction and $\langle 110\rangle$ the fast direction. ${ }^{46-48}$ The ratio of fast to slow directions is 
(a)
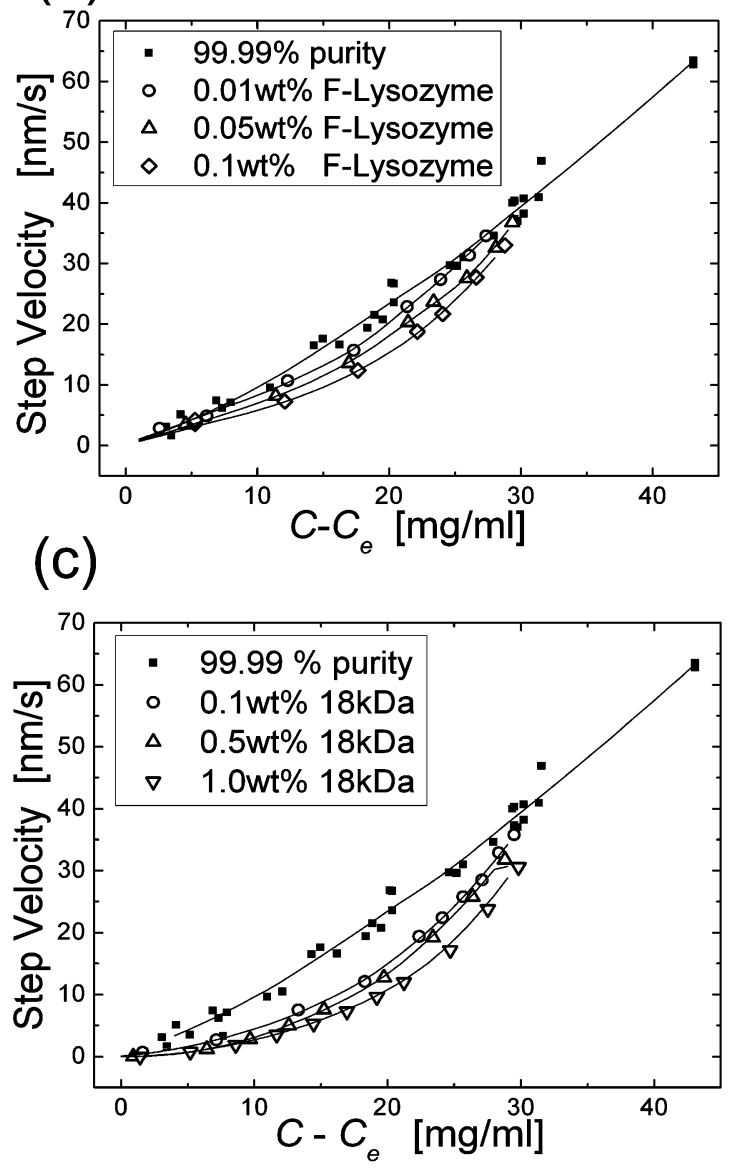

(b)

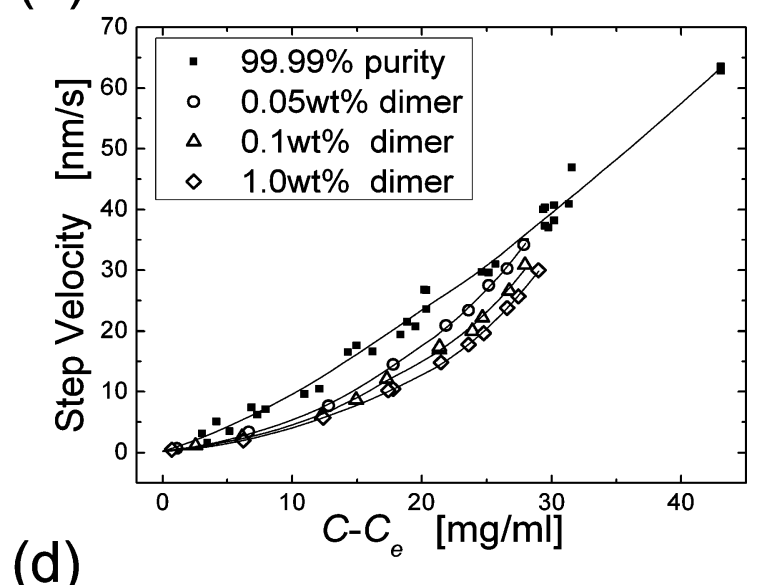

(d)

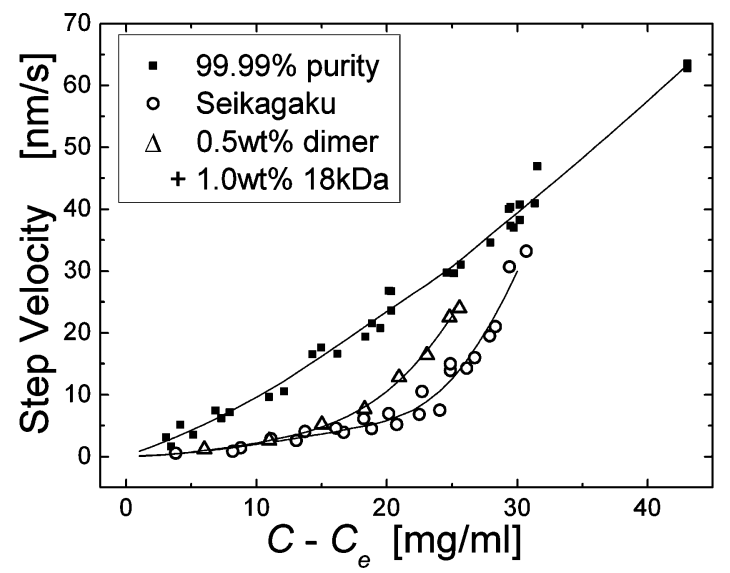

Figure 3. Step velocities in $\langle 110\rangle$ directions measured on $\{110\}$ faces of tetragonal lysozyme crystals under various supersaturations and protein impurity concentrations by LCM-DIM. All crystals were grown from $99.99 \%$ purity lysozyme solutions of $40 \mathrm{mg} / \mathrm{mL}$. Protein impurities added intentionally: (a) 0.01-0.1 wt \% F-lysozyme, (b) 0.05-1.0 wt \% dimer, (c) $0.1-1.0 \mathrm{wt} \% 18 \mathrm{kDa}$, and (d) $0.5 \mathrm{wt} \%$ dimer and $1.0 \mathrm{wt} \% 18 \mathrm{kDa}$. In (d), the result of Seikagaku lysozyme $\left(98.5 \%\right.$ purity) solutions of $40 \mathrm{mg} / \mathrm{mL}$ was also plotted. $C$ is a solute concentration and $C_{\mathrm{e}}$ the solubility. The data points were fitted using a local polynomial regression, Loess ${ }^{50,51}$ (solid curves). Other growth conditions: $25 \mathrm{mg} / \mathrm{mL} \mathrm{NaCl}$, in $50 \mathrm{mM}$ sodium acetate buffer $(\mathrm{pH} 4.5)$, at $18.0-26.0^{\circ} \mathrm{C}$.

$\sim 6$. In contrast, the $2 \mathrm{D}$ islands exhibit rounded tips in solutions containing F-lysozyme, dimer, and $18 \mathrm{kDa}$ added intentionally (Figure 2b-e) and in a Seikagaku lysozyme solution (Figure 2f). Their ratios of fast to slow directions (the ratios changed in the range of 3-5) become significantly smaller than that in the $99.99 \%$ purity solution. These results clearly indicate that the growth in the fast direction is more affected by impurities than that in the slow direction, as Dold and co-workers also reported. $^{10}$

In Figure 2, 0.1 wt \% F-lysozyme (b) shows weaker effects on the step morphology (less rounded 2D islands) than $0.5 \mathrm{wt}$ $\%$ dimer (c) and $1.0 \mathrm{wt} \% 18 \mathrm{kDa}(\mathrm{d})$, probably because of the lower impurity concentration: $0.1 \mathrm{wt} \%$ was the maximum concentration of F-lysozyme available in our experiments. In addition, the solution containing $0.5 \mathrm{wt} \%$ dimer and $1.0 \mathrm{wt} \%$ $18 \mathrm{kDa}(\mathrm{e})$ presents stronger effects on the step morphology than that containing only $0.5 \mathrm{wt} \%$ dimer (c) or $1.0 \mathrm{wt} \% 18$ $\mathrm{kDa}(\mathrm{d})$, because of the larger amount of protein impurities. However, note that Seikagaku lysozyme (f), which contains mainly $0.5 \mathrm{wt} \%$ dimer and $1.0 \mathrm{wt} \% 18 \mathrm{kDa}$ as impurities, ${ }^{40}$ shows significantly stronger effects (the ratio of fast to slow directions: $\approx 3$ ) than the $99.99 \%$ purity lysozyme with intentionally added $0.5 \mathrm{wt} \%$ dimer and $1.0 \mathrm{wt} \% 18 \mathrm{kDa}(\mathrm{e})$ (the ratio of fast to slow directions: $\approx 4$ ). These results indicate that trace amounts of other impurities included in Seikagaku lysozyme $e^{7,40}$ play a significant role.

Since the growth of elementary 2D islands in the fast direction $(\langle 110\rangle)$ is more affected by impurities than in the slow direction $(\langle 001\rangle)$, as shown in Figure 2, we measured step velocities in the fast direction (high kink density), with and without protein impurities under various supersaturations.

Figure 3 presents changes in step velocity as a function of supersaturation. As shown in Figure 3, even in the case of the 99.99\% purity solution without any impurities added intentionally (solid squares), the step velocity vs supersaturation plot shows a slightly concave shape, in particular under a low supersaturation range. Since kink density of a $2 \mathrm{D}$ island in the fast direction is high, nucleation of kinks (one-dimensional nucleation) cannot be the rate-determining step of the growth process. Hence, the concave shape of the step velocity vs supersaturation plot is most likely due to impurities present in the purified solution (>99.99\%). Vekilov and co-workers also observed impurity effects for a $99.99 \%$ pure lysozyme solution in forced flow experiments. ${ }^{49} \mathrm{We}$ reported previously that an impurity of $0.01 \mathrm{wt} \%$ significantly promotes heterogeneous $2 \mathrm{D}$ nucleation under a low supersaturation range. ${ }^{11}$ Figure 3 also demonstrates that all three protein impurities decreased the step velocity, as well as the case of Seikagaku lysozyme. In 

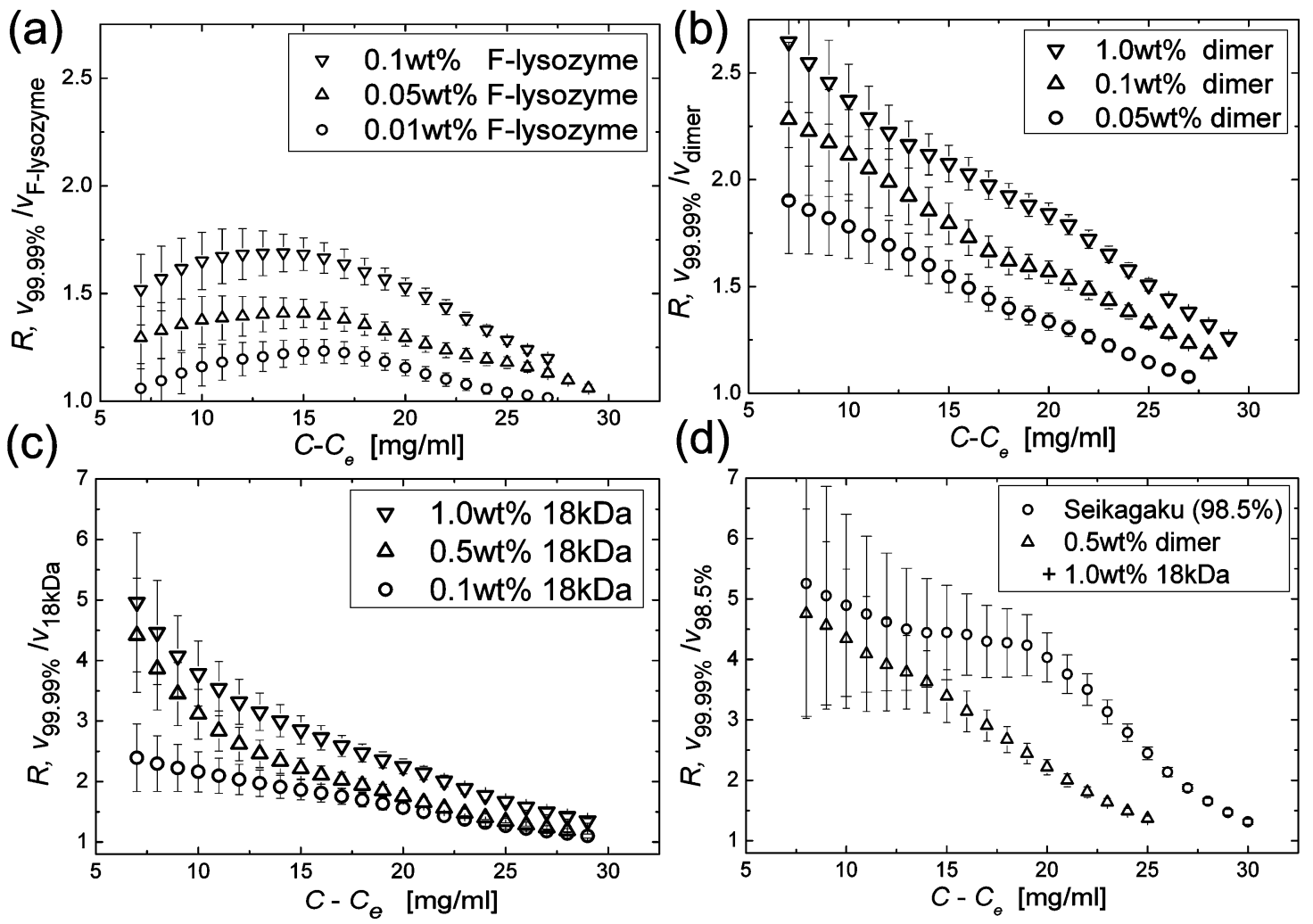

Figure 4. Changes in the ratios $R$ of the step velocity in a $99.99 \%$ purity solution, $v_{99.99 \%}$, to those in the solutions containing impurity, $v_{\mathrm{F}-1 y s o z y m e}$ $v_{\text {dimer }}, v_{\text {dimer }}$, and $v_{98.5 \%}$, as a function of supersaturation. $R$ was calculated from the fitted curves shown in Figure 3 , and the errors were evaluated from the standard error (90\% statistical significance) and the propagation of errors. All crystals were grown from $99.99 \%$ purity lysozyme solutions of $40 \mathrm{mg} / \mathrm{mL}$. Protein impurities added intentionally: (a) $0.01-0.1 \mathrm{wt} \%$ F-lysozyme, (b) $0.05-1.0 \mathrm{wt} \%$ dimer, (c) $0.1-1.0 \mathrm{wt} \% 18 \mathrm{kDa}$, and (d) $0.5 \mathrm{wt} \%$ dimer and $1.0 \mathrm{wt} \% 18 \mathrm{kDa}$. In (d), the results of Seikagaku lysozyme (98.5\% purity) solutions of $40 \mathrm{mg} / \mathrm{mL}$ were also plotted. $C$ is a solute concentration and $C_{\mathrm{e}}$ is the solubility. Other growth conditions: $25 \mathrm{mg} / \mathrm{mL} \mathrm{NaCl}$, in $50 \mathrm{mM}$ sodium acetate (pH 4.5$)$, at $18.0-26.0{ }^{\circ} \mathrm{C}$.

particular, the suppression of the step velocity by $18 \mathrm{kDa}$ and impurities included in the Seikagaku lysozyme (mainly $0.5 \%$ dimer and $1.0 \% 18 \mathrm{kDa})^{40}$ are significant. With increasing impurity concentration, the suppression of the step velocity increased, and the shape of the plots became more concave.

Although the effects of dimer on the growth kinetics of $\{110\}$ and $\{101\}$ faces of tetragonal lysozyme crystals have already been reported,,${ }^{91,24}$ to our knowledge this is the first study on the impurity effects of $18 \mathrm{kDa}$ on the growth kinetics of tetragonal lysozyme crystals. In most of the previous studies of impurity effects on lysozyme crystals, attention was only paid to dimer. However, our results clearly show that attention should be also paid to $18 \mathrm{kDa}$.

Under the experimental conditions adopted in this study, the strongest suppression of the step velocity, that is, strongest impurity effect, was found for Seikagaku lysozyme (open circles in Figure 3d). To simulate Seikagaku lysozyme we used $0.5 \mathrm{wt}$ $\%$ dimer and 1.0 wt \% $18 \mathrm{kDa}$ together with $99.99 \%$ purity lysozyme. However, the suppression of this solution (open triangles) was not as strong as that observed for Seikagaku lysozyme. This result implies that trace amounts of other impuritie(s) present in Seikagaku lysozyme contribute to the stronger suppression of the step velocity.

To evaluate the effects of these protein impurities quantitatively, we calculated the ratios $R$ of the step velocity in the $99.99 \%$ purity lysozyme solution, $v_{99.99 \%}$, to the step velocities in the solutions containing impurities, $v_{\mathrm{F}-\mathrm{lys} s z y m e}, v_{\text {dimer }}, v_{18 \mathrm{kDa}}$, and $v_{98.5 \%}$ at the same supersaturation. Thus, the ratio $R$ corresponds to the amount of the suppression of the step velocity by impurities compared to the case of the $99.99 \%$ purity solution. When the value of the ratio $R$ becomes unity, there is no suppression of the step velocity by impurities compared to that in the $99.99 \%$ purity solution. To calculate the ratio $R$, we fitted all the experimental data shown in Figure 3 with a local polynomial regression (Loess). ${ }^{50,51}$ The solid curves shown in Figure 3 demonstrate that the fitting curves represent well the supersaturation dependencies of the step velocities. Using the values obtained from the Loess fits, we calculated the ratio $R$ at the same supersaturation (Figure 4). Since in Figure 3 the typical error in the step velocity measurement at one supersaturation was about $1 \%$ for $99.99 \%$ purity lysozyme solutions and 3\% for Seikagaku lysozyme solutions (error bars fall inside of the symbols representing step velocities in Figure 3), the step velocity measurement was accurate enough. Hence, the error of the ratio $R$ mainly came from the local polynomial regression. The error bars shown in Figure 4 were calculated from the standard error (90\% statistical significance) coming from our fitting model and the propagation of errors.

As shown in Figure 4, the value of the ratio $R$ increased with increasing impurity concentration for all three protein impurities, showing stronger suppression of the step velocity with increasing impurity concentration. However, different protein impurities have different $R$ values and also show different supersaturation dependencies of $R$. Taking into account the large error of the ratio $R$ in the low supersaturation range (in particular, $C-C_{\mathrm{e}}<$ $10 \mathrm{mg} / \mathrm{mL}$ ), the differences in the values and shapes of the $R$ vs supersaturation plots are still significant. In the case of F-lysozyme (Figure 4a), the $R$ vs supersaturation plot tends to show a plateau at low supersaturation; that is, the value of $R$ does not show remarkable change taking into account large 


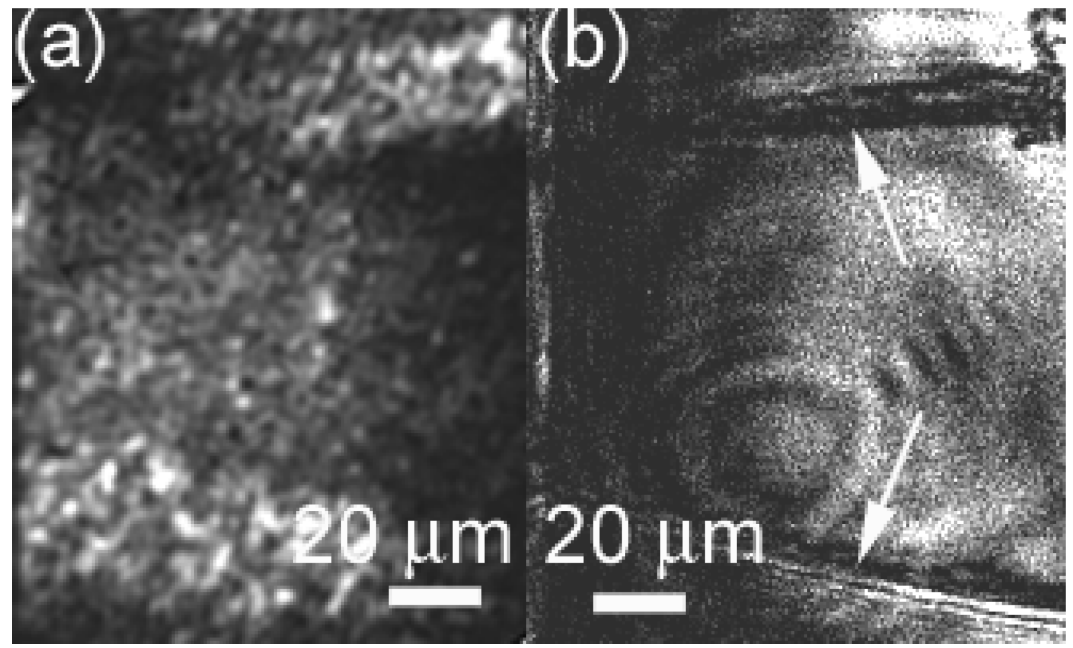

Figure 5. Adsorption sites of F-lysozyme on a $\{110\}$ surface of a tetragonal lysozyme crystal. The same field of view was observed by singlemolecule visualization of a thin-solution-layer type ${ }^{29,30}$ (a) and by LCM-DIM (b). (a) One bright dot corresponds to one F-lysozyme molecule adsorbed on the crystal surface. (b) Arrows show the positions of bunched steps on the crystal surface. Conditions: $99.99 \%$ purity lysozyme solution of $27 \mathrm{mg} / \mathrm{mL}, 0.1 \mathrm{nM}$ F-lysozyme, $25 \mathrm{mg} / \mathrm{mL} \mathrm{NaCl}$, in $50 \mathrm{mM}$ sodium acetate $(\mathrm{pH} 4.5)$, at $24.5{ }^{\circ} \mathrm{C}$.

errors of $R$ in this supersaturation range. Then $R$ starts decreasing in the supersaturation range of $12-17 \mathrm{mg} / \mathrm{mL}$. On the other hand, the $R$ vs supersaturation plots of dimer and $18 \mathrm{kDa}$ (Figure $4 \mathrm{~b}, \mathrm{c})$ exhibit a concave shape, and $R$ decreases with increasing supersaturation. In addition, the $R$ values of $18 \mathrm{kDa}$ are remarkably larger than those of F-lysozyme and dimer all through the supersaturation range. In the case of Seikagaku lysozyme (Figure 4d), the $R$ vs supersaturation plot shows a more complex shape: a significant shoulder is observed around a supersaturation of $C-C_{\mathrm{e}} \approx 18 \mathrm{mg} / \mathrm{mL}$. Furthermore, the solution containing $0.5 \%$ dimer and $1.0 \% 18 \mathrm{kDa}$ with $99.99 \%$ purity lysozyme could not reproduce the plot of Seikagaku lysozyme. For the same impurity the shape of $R$ vs supersaturation plots does not change regardless of the impurity concentration.

3.2. Adsorption Sites of Protein Impurities. To clarify the cause of the different dependencies of the ratio $R$ on supersaturation shown in Figure 4, we tried to observe the adsorption sites of fluorescent-labeled protein impurities on the crystal surface by single-molecule visualization of the thin-solutionlayer type. ${ }^{31,32}$ Figure 5a shows a typical single-molecule image: one bright dot corresponds, in principle, to one F-lysozyme molecule adsorbed on the crystal surface. Since the concentration of F-lysozyme was very dilute $(0.1 \mathrm{nM})$, the overlapping of diffusing F-lysozyme molecules on a crystal surface was avoided. ${ }^{31}$ However, once an F-lysozyme molecule was adsorbed (fixed) on a step, overlapping of fluorescence spots could potentially occur, because we cannot distinguish between two adsorbed molecules whose intermolecular distance is shorter than the resolution limit in lateral directions (in our case: 0.24 $\mu \mathrm{m})$. This overlapping could cause fluorescent dots with different intensities. Since the diffusion of F-lysozyme on a crystal surface was $4-5$ orders of magnitude slower than that in a bulk solution, ${ }^{31}$ we could visualize all F-lysozyme molecules appearing on a crystal surface. The image shown in Figure 5a was taken $2 \mathrm{~h}$ after F-lysozyme was introduced in the observation cell. During this period adsorption of F-lysozyme on a crystal surface proceeded, and then in Figure 5a most of F-lysozyme molecules ( $>95 \%$ ) visualized were fixed on the crystal surface.

Figure $5 \mathrm{~b}$ represents the same field of view observed by LCM-DIM, showing bunched steps (arrows) on the crystal surface. The comparison between these images clearly demonstrates the preferential adsorption of F-lysozyme on steps. Since the molecular weight of the fluorescent label is smaller than $3 \%$ of that of F-lysozyme, ${ }^{5}$ almost the entire molecular surface of F-lysozyme is the same as that of native lysozyme. Hence, F-lysozyme molecules preferentially adsorb on steps on the crystal surface as solute (native lysozyme) molecules, and are incorporated into a crystal at steps (more precisely, kinks on steps). With respect to the adsorption of F-lysozyme on the $\{110\}$ faces of the tetragonal lysozyme crystals our results were recently confirmed by Dai and co-workers. ${ }^{32}$

In the case of dimer, Nakada and co-workers revealed, by in situ AFM observation, that dimer molecules adsorb randomly on terraces of $\{101\}$ faces of tetragonal lysozyme crystals. ${ }^{9}$ However, for $\{110\}$ faces, no one has ever clarified the adsorption sites. To determine the adsorption sites of dimer on the $\{110\}$ face, we used F-dimer, since almost the complete molecular surface of F-dimer is expected to be the same as that of native dimer. Iimura and co-workers ${ }^{52}$ prepared dimer modified with a fluorescent label different from the one used in this study. They reported that incorporation rates of dimer and their F-dimer into a tetragonal lysozyme crystal do not differ. Hence, in this study it is reasonable to assume that the labeling of dimer molecules has no significant influence on the incorporation process. Figure 6a shows a typical single-molecule image: one bright dot corresponds, in principle, to one F-dimer molecule adsorbed on the crystal surface, although overlapping of F-dimer molecules could potentially occur as discussed in the case of F-lysozyme. Figure $6 \mathrm{~b}$ indicates the same field of view observed by LCM-DIM. The arrows show the positions of bunched steps on the crystal surface, and an arrowhead indicates the position of an elementary step. The comparison between Figure 6, panels a and b clearly indicates that F-dimer molecules adsorb randomly on terraces of the $\{110\}$ face, in contrast to the case of F-lysozyme.

Since dimer is composed of two covalently bonded lysozyme molecules, ${ }^{40}$ the bonding site between two monomeric lysozyme molecules and their orientation inside a dimer molecule differ from those between two neighboring lysozyme molecules in a crystal. ${ }^{53}$ Hence, a dimer molecule cannot be easily fitted into a kink site, at which a dimer molecule would suffer large steric hindrance and unfavorable bonding with the surroundings. In 


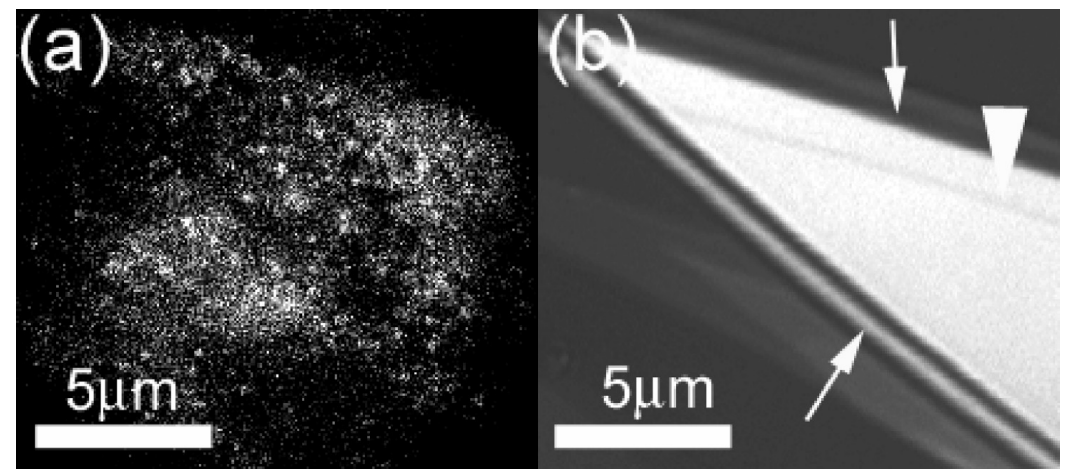

Figure 6. Adsorption sites of F-dimer on a $\{110\}$ surface of a tetragonal lysozyme crystal. The same field of view was observed by singlemolecule visualization of a thin-solution-layer type ${ }^{30}$ (a) and by LCM-DIM (b). (a) One bright dot corresponds to one F-dimer molecule adsorbed on the crystal surface. (b) Arrows show the positions of bunched steps on the crystal surface and an arrowhead indicates the position of an elementary step. Conditions: $99.99 \%$ purity lysozyme solution of $27 \mathrm{mg} / \mathrm{mL}, 0.1 \mathrm{nM} \mathrm{F}$-dimer, $25 \mathrm{mg} / \mathrm{mL} \mathrm{NaCl}$, in $50 \mathrm{mM}$ sodium acetate $(\mathrm{pH} 4.5)$, at $24.5^{\circ} \mathrm{C}$.

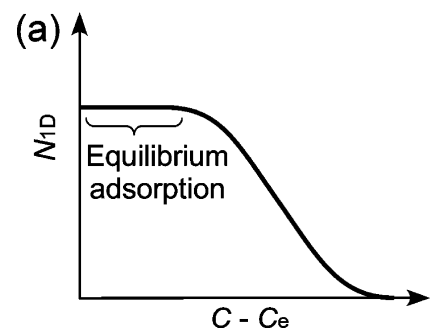

(b)

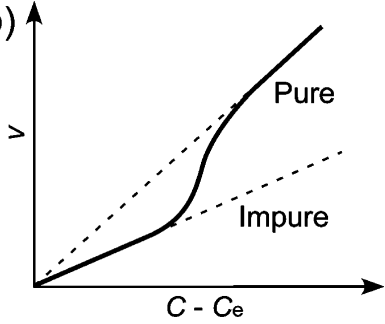

(c)

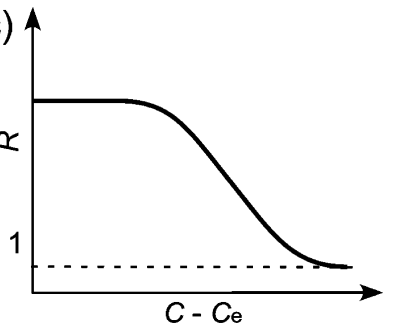

Figure 7. Schematic illustrations of impurity effects of F-lysozyme. Supersaturation dependencies of number density $N_{1 \mathrm{D}}[1 / \mathrm{m}]$ of adsorbed F-lysozyme on a step (a), step velocity $v$ (b) and the ratio $R$ (c). Using $\lambda_{0}$ (average distance between kinks) and $\lambda_{i}$ (average distance between free kinks), $N_{1 \mathrm{D}}$ is expressed as $N_{1 D}=1 / \lambda_{0}-1 / \lambda_{i}\left(0 \leq N_{1 D} \leq 1 / \lambda_{0}\right)$.

contrast, a terrace is spatially much more open than a kink site. Hence, a dimer molecule on a terrace would have a larger possibility to find a certain adsorption site, at which more favorable bonding can be formed than in the case of a dimer molecule at a kink site. The results shown in Figures 5 and 6 experimentally demonstrate, for the first time, that a microheterogeneous protein impurity molecule whose intermolecular bonding to the crystal surface is close to that of a solute molecule will preferentially adsorb on a step (i.e., kink), and that a microheterogeneous protein impurity molecule whose intermolecular bonding differs from that of a solute molecule will preferentially adsorb on different sites of the crystal surface.

3.3. Adsorption Sites and Supersaturation Dependencies of Step Velocities. From the difference in the adsorption sites of F-lysozyme and F-dimer on the $\{110\}$ faces of tetragonal lysozyme crystals, we attempted to explain the different dependencies of the ratio $R$ on supersaturation shown in Figure 4. Impurity models can be essentially divided into two groups. (I) Impurity molecules adsorbing at kink sites leading to kink blocking, which was first proposed by Bliznakov, ${ }^{54}$ and presented more extensively by Chernov. ${ }^{42}$ (II) Impurity molecules adsorbing on terraces (or steps) leading to step pinning, which was first described in the pioneer work of Cabrera and Vermileya. ${ }^{55}$ A more exhaustive overview of impurity models can be found in the work of Sangwal. ${ }^{56}$ We tried to explain our observations taking into account these two impurity models.

For F-lysozyme, steps (more precisely kinks on steps) are the major adsorption sites on the $\{110\}$ face. The movement of a growth ledge (i.e., step) is ensured simultaneously by different rates of attachment of growth entities at incorporation sites (kinks) occupied and unoccupied with impurities. ${ }^{57}$ Under a low supersaturation range, F-lysozyme molecules have enough time for adsorption because of slow advancement of steps. Hence, in this supersaturation range, it is reasonable to assume that the adsorption and desorption of F-lysozyme molecules on a step (i.e., kinks) reach equilibrium: that is, the ratio of a number of occupied kinks by impurity to a total number of kinks on a step becomes constant irrespective of supersaturation, ${ }^{58}$ as schematically shown in Figure 7a. Considering the high kink density in the $\langle 110\rangle$ direction, the step kinetic coefficient $\beta$ can be assumed to be proportional to the kink density: ${ }^{42}$

$$
\beta=a v\left(a / \lambda_{0}\right) \exp (-E / k T)
$$

where $a$ is the size of an attaching species in a lattice, $v$ is the frequency of thermal vibrations of a molecule in a crystal and a solution, $\lambda_{0}$ is the average distance between kinks, and $E$ is the activation potential barrier for a solute molecule to be incorporated into a kink. Now, if an impurity, which poisons kinks, is present in a solution the kinetic coefficient is assumed to be proportional to the density of free kinks: ${ }^{42}$

$$
\beta\left(C_{\mathrm{imp}}\right)=\beta_{0} \lambda_{0} / \lambda_{i}
$$

where $C_{\text {imp }}$ is the impurity concentration and $\lambda_{i}$ the average distance between free kinks. Taking into account eq 1, it becomes clear that at a fixed impurity concentration a decrease in the kinetic coefficient will lead to a linear decrease in the step velocity. ${ }^{42}$

Under a low supersaturation range, since the ratio of a number of occupied kinks by impurity to a total number of kinks on a step is constant (i.e., equilibrium adsorption), the $v$ vs $C-C_{\mathrm{e}}$ 

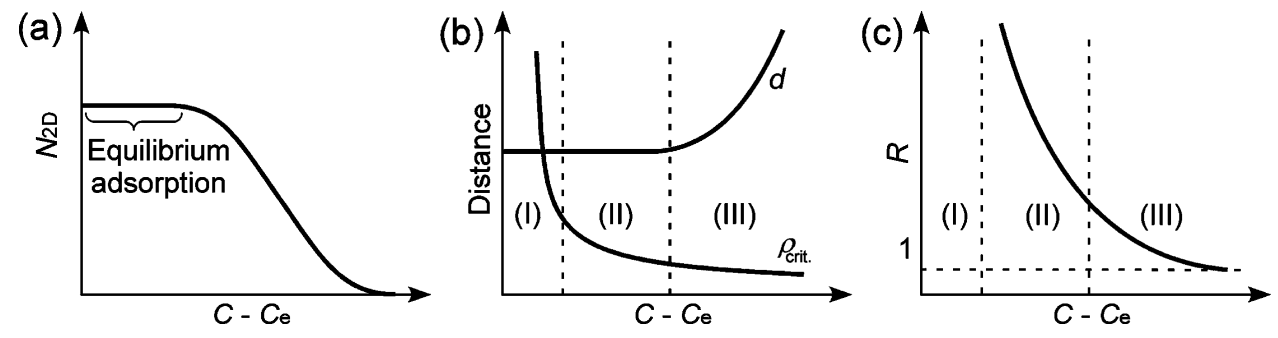

Figure 8. Schematic illustrations of impurity effects of dimer. Supersaturation dependencies of number density $N_{2 \mathrm{D}}\left[1 / \mathrm{m}^{2}\right]$ of adsorbed dimer on a terrace (a), $d$ (distance between adjacent dimer molecules adsorbed on a terrace) and $\rho_{\text {crit }}$ (radius of a critical 2D nucleus) (b) and the ratio $R$ (c). Here $d$ is calculated as $d=1 / \sqrt{N_{2 \mathrm{D}}}$.

plot follows a line (named "impure" in Figure 7b) with a constant slope, whose kinetic coefficient is smaller than in the case of a pure sample.

With increasing supersaturation, the increase in step velocity decreases the exposure time of adsorption sites to impurities in a solution, ${ }^{58}$ and this tends to decrease the impurity effects (i.e., less kink sites are blocked by impurity molecules). Hence, in a middle supersaturation range, with increasing supersaturation the ratio of the number of occupied kinks by impurity to the total number of kinks on a step decreases and finally reaches zero under a high supersaturation range (Figure 7a). Therefore, under a high supersaturation range, $v$ vs $C-C_{\mathrm{e}}$ plot follows the line that is observed in the case of pure sample (named "pure" in Figure $7 \mathrm{~b}$ ). Under a middle supersaturation range, the transition of the $v$ vs $C-C_{\mathrm{e}}$ plot from the impure line to the pure one is observed. According to such a picture, the phenomena that we observed can be explained as follows (Figure 7c): under a low supersaturation range the degree of impurity effect (the ratio $R$ ) remains constant with increasing supersaturation, and in the middle and high supersaturation ranges $R$ decreases with increasing supersaturation.

So far we did not find any experimental evidence for pinning of step advancement by F-lysozyme adsorbed at kinks. As discussed in the above paragraphs, we explained the supersaturation dependency of $R$ without taking into account the pinning of step advancement. Since most of the molecular surface of F-lysozyme is the same as that of native lysozyme, we suppose that F-lysozyme adsorbed at kinks would not show significant pinning of steps. To clarify this issue, we have to simultaneously observe the adsorption process of individual F-lysozyme molecules on a step and the subsequent response of a step. We are planning to do such type of observation in the near future.

In the case of dimer, terraces are the major adsorption sites on the $\{110\}$ faces, as shown in Figure 6. Thus, to describe the impurity effects of dimer on step advancement, we follow the step pinning model ${ }^{55}$ which analyzes the impurity effect by comparing the critical curvature of a step to the average spacing of adsorbed impurities.

Under a low supersaturation range, dimer would show equilibrium adsorption on a terrace, as schematically shown in Figure 8a. In Figure 4b, we did not observe a plateau under a low supersaturation range, where $R$ remained constant. This result can be satisfactorily explained by taking into account the supersaturation dependency of the radius of a critical nucleus. Assuming a circular 2D nucleus with a double layer, ${ }^{11}$ its critical radius $\rho_{\text {crit }}$ can be expressed as

$$
\rho_{\text {crit }}=s \kappa /(2 \Delta \mu)
$$

where $\Delta \mu$ is the chemical potential difference between molecules in a crystal and a solution, $s$ is the area one molecule occupies inside a nucleus, and $\kappa$ is the ledge free energy of a 2D cluster. Hence, with increasing supersaturation $\rho_{\text {crit }}$ decreases, as schematically shown in Figure 8b. On the other hand, distance $d$ between adjacent dimer molecules adsorbed on a terrace can be expressed as

$$
d=1 / \sqrt{N_{2 \mathrm{D}}}
$$

where $N_{2 \mathrm{D}}$ is the number of dimer molecules adsorbed per unit area. When $d<2 \rho_{\text {crit }}$ steps stop advancing (supersaturation range I in Figure $8 \mathrm{~b}$ ): in this region $R$ cannot be defined. However, in the supersaturation range II (Figure 8b) where still equilibrium adsorption of dimer exists, with increasing supersaturation a decrease in $\rho_{\text {crit }}$ results in a decrease of the impurity effect (i.e., $R$ ). In the supersaturation range III, the decrease in $\rho_{\text {crit }}$ and the increase in $d$ decrease the impurity effect with increasing supersaturation. The supersaturation range observed in Figure $4 \mathrm{~b}$ would correspond to ranges II and III in Figure 8c.

For $18 \mathrm{kDa}$, we observed a very similar dependency of $R$ on supersaturation to that observed for dimer (Figure $4 b, c)$. Taking into account that the intermolecular bonding between $18 \mathrm{kDa}$ and a crystal surface is different from that in the case of a native lysozyme molecule, it seems reasonable to assume that $18 \mathrm{kDa}$ also adsorbs randomly on terraces as in the case of dimer.

The impurity effects observed for Seikagaku lysozyme were more complex (Figure 4d), and could not be reproduced by the solution containing $0.5 \mathrm{wt} \%$ dimer and $1.0 \mathrm{wt} \% 18 \mathrm{kDa}$, although these two are the main impurities included in Seikagaku lysozyme. ${ }^{40}$ This result indicates that trace amounts of other impure molecules present in Seikagaku lysozyme play a significant role: one possibility is a $39 \mathrm{kDa}$ polypeptide, ${ }^{40}$ and another, less likely possible, is the presence of two very large lysozyme oligomers $(1700 \mathrm{kDa}$ and $320 \mathrm{kDa}){ }^{7}$

Recent progress in advanced optical microscopy enabled us to observe in situ elementary growth steps and individual protein molecules in the same field of view. Such molecular level in situ observations provided the first direct evidence that adsorption sites of impurities play an important role in impurity effects. The techniques used in this study can be a promising means to obtain comprehensive understanding on mechanisms of impurity effects on macromolecule crystallization. Although the explanations in this study are still qualitative, a quantitative model will be presented in the near future.

\section{Conclusions}

We studied the effects of protein impurities (F-lysozyme, dimer, $18 \mathrm{kDa}$, and impurities present in Seikagaku lysozyme) on the step velocity of elementary $2 \mathrm{D}$ islands on the $\{110\}$ 
faces of tetragonal lysozyme crystals under various supersaturations and impurity concentrations utilizing LCM-DIM. We also observed in situ individual molecules of F-lysozyme and F-dimer adsorbed on the $\{110\}$ faces. From these direct and noninvasive in situ observations we found the following key results:

(1) Different protein impurities exhibited different dependencies of the suppression of the step velocities on supersaturation. The $R$ vs supersaturation plots of F-lysozyme showed first a plateau at low supersaturation and then a decrease at higher supersaturations, whereas the plots of dimer and $18 \mathrm{kDa}$ presented a concave shape. The impurity effect of $18 \mathrm{kDa}$ was significantly stronger than that of dimer, although so far much less attention was paid to $18 \mathrm{kDa}$. The $R$ vs supersaturation plots of Seikagaku lysozyme exhibited a complex shape, which could not be reproduced by $99.99 \%$ purity lysozyme and two major impurities (dimer and $18 \mathrm{kDa}$ ), suggesting that trace amounts of impurities $(<0.01 \%)$ in Seikagaku lysozyme play a crucial role.

(2) Single-molecule visualization and LCM-DIM observations of the same field of view revealed that F-lysozyme adsorbed preferentially on steps, whereas F-dimer adsorbed randomly on terraces. This indicates that microheterogeneous impurity molecules whose intermolecular bonding with a crystal surface is close to that of solute molecules adsorb preferentially on steps (i.e., kinks), and that microheterogeneous impurity molecules whose intermolecular bonding differs from that of solute molecules adsorb on different sites of a crystal surface.

(3) We could successfully explain the $R$ vs supersaturation plots of F-lysozyme and dimer, using different adsorption sites of these protein impurities on the crystal surface and taking into account the kink blocking and step pinning impurity models.

(4) The $R$ vs supersaturation plots of $18 \mathrm{kDa}$ similar to those of dimer suggest that $18 \mathrm{kDa}$ molecules also adsorb randomly on terraces.

Acknowledgment. The authors are grateful for the partial support by Grants-in-Aid (Nos. 17034007 and 18360003) of Scientific Research of the Ministry of Education, Science and Culture Japan (G.S.), the support by Grant No. ESP 2006-11327 of the Ministry of Education and Science (MEC), Spain, and the Consolider-Ingenio 2010 project "Factoría Española de crystalización" (F.O., J.G., and A.V.D.). This work has been carried out as a part of Project Research B in the Center for Interdisciplinary Research, Tohoku University, as well as "Ground-based Research Announcement for Space Utilization" promoted by the Japan Space Forum.

\section{References}

(1) Vekilov, P. G.; Rosenberger, F. J. Cryst. Growth 1996, 158, 540551.

(2) Hirschler, J.; Fontecilla-Camps, J. C. J. Cryst. Growth 1997, 171, 559565.

(3) Judge, R. A.; Forsythe, E. L.; Pusey, M. L. Biotechnol. Bioeng. 1998, $59,776-785$

(4) Thomas, B. R.; Carter, D.; , F.; Rosenberger, F. J. Cryst. Growth 1998, 187, 499-510.

(5) Matsui, T.; Sazaki, G.; Hondoh, H.; Matsuura, Y.; Nakada, T.; Nakajima, K. J. Cryst. Growth 2006, 293, 415-422.

(6) Sazaki, G.; Aoki, S.; Ooshima, H.; Kato, J. J. Cryst. Growth 1994, 139, 95-1003.

(7) Thomas, B. R.; Vekilov, P. G.; Rosenberger, F. Acta Crystallogr., Sect. D: Biol. Crystallogr. 1998, 54, 226-236.

(8) Burke, M. W.; Leardi, R.; Judge, R. A.; Pusey, M. L. Cryst. Growth Des. 2001, 1, 333-337.
(9) Nakada, T.; Sazaki, G.; Miyashita, S.; Durbin, S. D.; Komatsu, H. J. Cryst. Growth 1999, 196, 503-510.

(10) Dold, P.; Ono, E.; Tsukamoto, K.; Sazaki, G. J. Cryst. Growth 2006, 293, 102-109.

(11) Van Driessche, A. E. S.; Sazaki, G.; Otalora, F.; Gonzalez-Rico, F. M.; Dold, P.; Tsukamoto, K.; Nakajima, K. Cryst. Growth Des. 2007, 7, 1980-1987.

(12) Abergel, C.; Nesa, M. P.; Fontecilla-Camps, J. C. J. Cryst. Growth 1991, 110, 11-19.

(13) Hirschler, J.; Fontecilla-Camps, J. C. Acta Crystallogr., Sect. D: Biol.Crystallogr 1996, 52, 806-812.

(14) Ewing, F. L.; Forsythe, E. L.; van der Woerd, M.; Pusey, M. L. J. Cryst. Growth 1996, 160, 389-397.

(15) Bhamidi, V.; Hanson, B. L.; Edmundson, A.; Skrzypczak-Jankun, E.; Schall, C. J. Cryst. Growth 1999, 204, 542-552.

(16) Caylor, C.; Dobrianov, I.; Lemay, S. G.; Kimmer, C.; Kriminski, S.; Finkelstein, K. D.; Zipfel, W.; Webb, W. W.; Thomas, B. R.; Chernov, A. A.; Thorne, R. E. Proteins: Struct., Funct., Genet. 1999, 36, 270281.

(17) Dobrianov, I.; Caylor, C.; Lemay, S. G.; Finkelstein, K. D.; Thorne, R. E. J. Cryst. Growth 1999, 196, 511-523.

(18) Carter, D. C.; Lim, K.; Ho, J. X.; Wright, B. S.; Twigg, P. D.; Miller, T. Y.; Chapman, J.; Keeling, K.; Ruble, J.; Vekilov, P. G.; Thomas, B. R.; Rosenberger, F.; Chernov, A. A. J. Cryst. Growth 1999, 196, 623-637.

(19) Snell, E. H.; Judge, R. A.; Crawford, L.; Forsythe, E. L.; Pusey, M. L.; Sportiello, M.; Todd, P.; Bellamy, H.; Lovelace, J.; Cassanto, J. M.; Borgstahl, G. E. O. Cryst. Growth Des. 2001, 1, 151-158.

(20) Robert, M. C.; Capelle, B.; Lorber, B.; Giege, R. J. Cryst. Growth 2001, 232, 489-497.

(21) Yoshizaki, I.; Kadowaki, A.; Iimura, Y.; Igarashi, N.; Yoda, S.; Komatsu, H. J. Synchrotron Rad. 2004, 11, 30-33.

(22) Moreno, A.; Theobald-Dietrich, A.; Lorber, B.; Sauter, C.; Giege, R. Acta Crystallogr., Sect. D: Biol. Crystallogr. 2005, 61, 789-792.

(23) Hondoh, H.; Nakada, T. J. Cryst. Growth 2005, 275, e1423-e1429.

(24) Yoshizaki, I.; Fukuyama, S.; Koizumi, H.; Tachibana, M.; Kojima, K.; Matsuura, Y.; Tanaka, M.; Igarashi, N.; Kadowaki, A.; Rong, L.; Adachi, S.; Yoda, S.; Komatsu, H. J. Cryst. Growth 2005, 290, 185191.

(25) Yau, S.-T.; Thomas, B. R.; Vekilov, P. G. Phys. Rev. Lett. 2000, 85, 353-356.

(26) Yau, S.-T.; Thomas, B. R.; Galkin, O.; Gliko, O.; Vekilov, P. G. Proteins: Struct., Funct., Genet. 2001, 43, 343-352.

(27) Funatsu, T.; Harada, Y.; Tokunaga, M.; Saito, K.; Yanagida, T. Nature 1995, 374, 555-559.

(28) Sako, Y.; Uyemura, T. Cell Struct. Funct. 2002, 27, 357-365.

(29) Cognet, L.; Coussen, F.; Choquet, D.; Lounis, B. C. R. Phys. 2002, $3,645-656$

(30) Wazawa, T.; Ueda, M. Adv. Biochem. Eng. Biotechnol. 2005, 95, 77106.

(31) Sazaki, G.; Okada, M.; Matsui, T.; Watanabe, T.; Higuchi, H.; Tsukamoto, K.; Nakajima, K. Cryst. Growth Des. 2008, 8, 2024-2031.

(32) Dai, G.; Sazaki, G.; Matsui, T.; Tsukamoto, T.; Nakajima, K.; Kang, Q.; Hu, W. J. Am. Chem. Soc., submitted.

(33) Van Driessche, A. E. S.; Otálora, F.; Gavira, J. A.; Sazaki, G. Cryst. Growth Des. 2008, 9, 4316-4323.

(34) Tsukamoto, K.; Yai, S.; Sazaki, G. J. Jpn. Assoc. Cryst. Growth 2003, 30, 81 (in Japanese).

(35) Yai, S. Graduation thesis, Tohoku University, 2003.

(36) Sazaki, G.; Matsui, T.; Tsukamoto, K.; Usami, N.; Ujihara, T.; Fujiwara, K.; Nakajima, K. J. Cryst. Growth 2004, 262, 536-542.

(37) Sazaki, G.; Tsukamoto, K.; Yai, S.; Okada, M.; Nakajima, K. Cryst. Growth Des. 2005, 5, 1729-1735.

(38) Suzuki, Y.; Sazaki, G.; Matsui, T.; Nakajima, K.; Tamura, K. J. Phys. Chem. B 2005, 109, 3222-3226.

(39) Dold, P.; Nishimura, Y.; Van Driessche, A. E. S.; Tsukamoto, K.; Sazaki, G. to be submitted.

(40) Thomas, B. R.; Vekilov, P. G.; Rosenberger, F. Acta Crystallogr., Sect. D: Biol.Crystallogr. 1996, 52, 776-784.

(41) Sazaki, G.; Kurihara, K.; Nakada, T.; Miyashita, S.; Komatsu, H. J. J. Cryst. Growth 1996, 169, 355-360.

(42) Chernov, A. A. Modern Crystallography, Vol. III: Growth of Crystals; Springer-Verlag: Berlin, 1984.

(43) Nadarajah, A.; Pusey, M. L. Acta Crystallogr., Sect. D: Biol. Crystallogr. 1996, 52, 983-996.

(44) Van Driessche, A. E. S.; Otálora, F.; Sazaki, G.; Gavira, J. A. Cryst. Growth Des. 2008, 8, 3623-3629.

(45) McPherson, A. Crystallization of Biological Macromolecules; Cold Spring Harbor Laboratory Press: New York, 1999. 
(46) Durbin, S. D.; Feher, G. J. Mol. Biol. 1990, 212, 763-774.

(47) Durbin, S. D.; Carlson, W. E. J. Cryst. Growth 1992, 122, 71-79.

(48) Li, M.; Nadarajah, A.; Pusey, M. L. Acta Crystallogr., Sect. D: Biol. Crystallogr. 1999, 55, 1036-1045.

(49) Vekilov, P. G.; Rosenberger, F. J. Cryst. Growth 1998, 186, 251261.

(50) Cleveland, W. S. J. Am. Stat. Assoc. 1979, 74, 829-836.

(51) Cleveland, W. S.; Devlin, S. J. J. Am. Stat. Assoc. 1988, 83, 596610.

(52) Iimura, Y.; Yoshizaki, I.; Nakamura, H.; Yoda, S.; Komatsu, H. Jpn J. Appl. Phys. 2003, 42, 5831-5836.

(53) Matsuura, Y.; Chernov, A. A. Acta Crystallogr., Sect. D: Biol.Crystallogr. 2003, 59, 1347-1356.
(54) Bliznakov, G. Fortschr. Min. 1958, 36, 149-191.

(55) Cabrera, N.; Vermilyea, D. A. Growth and Perfection of Crystals, In Growth and Perfection of Crystals, Proceedings; Doremus, R. H., Roberts, B. W., Turnbull, D., Eds.; John Wiley \& Sons: New York, 1958; pp 393-410.

(56) Sangwal, K. Additives and Crystallization Processes: From Fundamentals to Applications; John Wiley \& Sons: West Sussex, 2007.

(57) Sangwal, K. Prog. Cryst. Growth Charact. 1996, 32, 3-43.

(58) van der Eerden, J. P. In Handbook of Crystal Growth 1: Fundamentals; Hurle, D. T. J., Ed.; North-Holland: Amsterdam, 1993; Vol. 1a, pp $307-479$.

\section{CG8006684}

